\title{
ANÁLISE DO DESEMPENHO MOTOR E DO EQUILÍBRIO CORPORAL DE IDOSOS ATIVOS COM HIPERTENSÃO ARTERIAL E DIABETES TIPO 2
}

\author{
ANALYSIS OF MOTOR PERFORMANCE AND CORPORAL BALANCE OF ACTIVE ELDERLY WITH \\ ARTERIAL HYPERTENSION AND TYPE 2 DIABETES
}

\section{Camila Monteiro Agostinia, Vinícius Santos Rodrigues ${ }^{b}$, Andrea Carmen Guimarães ${ }^{c}$, Laila Cristina Moreira Damázio ${ }^{\mathrm{d}}$, Nathália Nascimento Vasconcelos ${ }^{\mathrm{e}}$}

acah_agostini@yahoo.com.br, bviniciushp95@gmail.com, candreaguimaraes@ufsj.edu.br, dlailacmd@gmail.com, enath_nv@ufsj.edu.br Universidade Federal de São João del-Rei - São João del-Rei (MG), Brasil

Data de recebimento do artigo: 01/08/2017 Data de aceite do artigo: 03/10/2017

\section{RESUMO}

Introdução: O Brasil está em processo de envelhecimento populacional. Os idosos apresentam alteraçôes neuromusculares e prevalência de doenças crônicas. A prática de exercícios físicos pode minimizar alterações e patologias associadas ao envelhecimento. Objetivo: Avaliar o equilíbrio corporal e o desempenho motor de idosos hipertensos e com diabetes tipo 2. Métodos: Estudo transversal realizado com 54 pacientes (48 mulheres e 6 homens), sendo 51 hipertensos, 16 diabéticos e 13 hipertensos e diabéticos. Utilizouse a Escala de Berg para avaliação do equilíbrio corporal, e o teste manual de força muscular (TMF) para avaliação da força muscular. Para avaliar amplitude de movimento articular (ADM), utilizou-se o goniômetro manual. Os testes de Thomas, de isquiotibiais e de reto femoral foram utilizados para avaliação de encurtamentos musculares, e o protocolo de Cerny, para avaliação da velocidade e cadência da marcha. Resultados: $\mathrm{O}$ equilíbrio dos pacientes diabéticos, em média, foi de 49,31+10,85 pontos, e o dos pacientes hipertensos, $53,13+6,21$ pontos. Os valores da cadência da marcha demonstraram diferença significativa entre os grupos, sendo 119,44 passos/minuto para pacientes hipertensos e 108,5 passos/ minuto para diabéticos. As diferenças de tempo e velocidade da marcha não foram significativas. Os dados da força muscular demonstraram valores acima de 3 pontos no TMF. Conclusóes: Pacientes diabéticos apresentaram pior desempenho na marcha e equilíbrio corporal, numa comparaçáo com hipertensos. Um programa de atividade física é estratégia eficaz para aumento da força muscular e para melhora do equilíbrio corporal e de parâmetros da marcha.

Palavras-chave: Idoso; equilíbrio postural; hipertensão arterial; diabetes mellitus; condicionamento físico humano.

\section{ABSTRACT}

Introduction: Brazil is in the process of demographic aging. The elderly have neuromuscular disorders and high prevalence of chronic diseases. Physical exercises can minimize changes and conditions associated with aging. Objective: To evaluate the corporal balance and motor performance of elderly with arterial hypertension and type 2 diabetes. Methods: A Cross-sectional study with 54 patients (48 women and 6 men), 51 hypertensives patients, 16 diabetics and 13 hypertensives and diabetics. The Berg balance scale was used to evaluate body balance, and muscle strength manual test (TMF) was used to evaluate muscle strength. In the evaluation of amplitude motion (AM), the manual goniometer was used. Tests of Thomas, hamstrings and rectus femoris were used for evaluation of muscle shortening, and Cerny protocol was used to evaluate velocity and cadence. Results: The body balance of diabetic patients was, on average, $49.31+10.85$ points, and of hypertensive patients, $53.13+6.21$ points. The gait cadence values showed significant differences between groups, with 119.44 steps/minute for hypertensive patients and 
108.5 steps/minute for diabetics. The differences in time and walking velocity were not significant. The data of muscle strength showed values above 3 points in the TMF. Conclusions: Diabetic patients had poorer performance in gait and corporal balance, on a comparison with hypertensives. A program of physical activity is an effective strategy to increases strength and improves corporal balance and gait parameters.

Keywords: Elderly; postural balance; hypertension; diabetes mellitus; human fitness.

\section{Introdução}

O Brasil está passando por um processo de envelhecimento populacional, o que resulta no crescimento do número de idosos no país. De acordo com a projeção de população mais recente, realizada pelo Instituto Brasileiro de Geografia e Estatística (IBGE), o grupo de pessoas com 60 anos ou mais de idade terá acentuado aumento na participação relativa da população, passando de $13,8 \%$, em 2020 , para $33,7 \%$, em $2060^{1}$. A população idosa apresenta um declínio no desempenho do sistema neuromuscular, com perda da massa muscular, redução da flexibilidade, da força, da resistência e da mobilidade articular, limitando a capacidade de coordenação e do equilíbrio corporal ${ }^{2}$.

O envelhecimento ocasiona um decréscimo natural de massa magra, perdas de massa óssea, diminuição de tecido muscular esquelético e de água corporal ${ }^{2}$. A diminuição da massa muscular é a principal responsável pelo comprometimento na mobilidade e na capacidade funcional dos idosos, gerando consequências negativas na marcha e no equilíbrio, aumentando o risco de queda e de perda da independência funcional ${ }^{2}$.

Os idosos apresentam alta prevalência de doenças crônicas. Segundo dados da Pesquisa Nacional de Saúde (PNS), realizada entre agosto de 2013 e fevereiro de 2014, a proporção de hipertensos no Brasil é de 44,4\% entre pessoas de 60 a 64 anos, $52,7 \%$ entre pessoas de 65 e 74 anos e $55 \%$ nas pessoas com mais de 75 anos. Já com relação ao diabetes, a proporção é de $14,5 \%$ entre 60 e 64 anos, $19,9 \%$ entre 65 e 74 anos e $19,6 \%$ para 75 anos ou mais ${ }^{3}$.

As doenças crônicas degenerativas ou não transmissíveis (Dant) constituem um sério problema de saúde pública, sendo responsáveis por $63 \%$ das mortes no mundo ${ }^{4}$. No Brasil, essas doenças são responsáveis por mais de $70 \%$ das mortes ${ }^{3}$.

A atividade física pode ser entendida como qualquer movimento voluntário produzido pela musculatura esquelética que leve a um gasto energético ${ }^{5}$. Um dos principais benefícios dessa prática é a manutenção da capacidade funcional (que é a condição para se executar atividades da vida diária de maneira autônoma, como tomar banho, vestir-se e levantar-se), principalmente para os idosos ${ }^{6}$.
Pesquisas indicam que a atividade física diminui o risco de doenças crônicas degenerativas, além de aumentar o bem-estar ${ }^{7}$. É estimado que a inatividade física e a dieta estão relacionadas a $75 \%$ dos casos novos de doenças não transmissíveis, sendo que o baixo condicionamento cardiorrespiratório, a pouca força muscular e o sedentarismo aumentam de três a quatro vezes a prevalência da Síndrome Metabólica ${ }^{8}$.

O exercício físico contribui para o aumento da força muscular do idoso, favorecendo a diminuição do risco de quedas, melhorando o equilíbrio e a realização das atividades de vida diária ${ }^{9}$. A prática de exercícios físicos promove melhora na aptidão física, que pode ser definida como a capacidade de o indivíduo apresentar desempenho físico adequado em suas atividades da vida diária, evitando o cansaço precoce ${ }^{10}$. A aptidão cardiorrespiratória, a flexibilidade, a força e a resistência muscular são os componentes da aptidão física relacionados à saúde que podem ser mais influenciados pelas atividades físi$\operatorname{cas}^{11}$. A realização de exercícios físicos previne e desacelera as perdas funcionais do envelhecimento, além de auxiliar na prevenção e tratamento de doenças crônicas e outras patologias, sendo recomendada para a melhora do estado de saúde ${ }^{12}$.

A prática de exercício físico promove benefícios significativos à saúde da populaçẫo. Manter-se ativo promove benefícios que contribuem para a independência funcional e, consequentemente, para a melhora da qualidade de vida ${ }^{13}$. Desse modo, o exercício físico contribui para a redução dos gastos na saúde pública, pois ajuda a prevenir e a tratar as consequências do sedentarismo, além de diminuir a morbidade e a mortalidade da população ${ }^{14}$.

\section{Objetivo}

O objetivo do estudo é avaliar o desempenho motor e o equilíbrio de idosos com hipertensão arterial e diabetes tipo 2 que praticam atividade física no Laboratório de Pesquisa e Intervenção Psicossocial (Lapip) do Departamento de Psicologia da Universidade Federal de São João del Rei (UFSJ), com intuito de quantificar os benefícios que o programa de exercícios vem proporcionando aos envolvidos. 


\section{Métodos}

Foi realizado um estudo com delineamento transversal entre março e dezembro de 2015, no Lapip. Criado em junho de 2000 para a Unidade Curricular de Educação Física Adaptada, esse laboratório tem se desenvolvido de forma relevante na área de pesquisa e extensão, além de possuir amplo atendimento a usuários externos por meio de projetos com grupos, instituiçóes e comunidades em São João del Rei.

Foram avaliados 54 pacientes de ambos os sexos, sendo 51 hipertensos, 16 diabéticos e, desses, 13 hipertensos e diabéticos. A idade média dos participantes foi de $61,74+8,62$ para os hipertensos e $60,75+8,72$ para os diabéticos.

Para avaliação das amplitudes de movimento (ADM) foi utilizado um goniômetro universal da marca Carci na análise do movimento ativo do paciente ${ }^{15}$. Foram avaliadas as goniometrias de flexão e extensão do joelho, plantiflexão e dorsiflexão do tornozelo, flexão e extensão do quadril, flexão e extensão do ombro, flexão e extensão do cotovelo e flexão e extensão do punho.

Para avaliar a força muscular dos músculos flexores e extensores de punho e dedos, tríceps braquial, bíceps braquial, peitoral maior, isquiotibiais, tibial anterior, tríceps sural e quadríceps, foi utilizado como referência o teste manual de força muscular, que possui cinco níveis: 0 ) ausência de contração muscular; 1) contração muscular, sem movimento articular; 2) movimento articular sem atuação da gravidade; 3) movimento contra a gravidade; 4) movimento contra uma pequena resistência; 5) movimento contra grande resistência ${ }^{16}$.

O equilíbrio corporal dos pacientes foi mensurado utilizando a escala de Berg, um teste contendo catorze tarefas comuns nas atividades de vida diárias, para avaliação do equilíbrio estático e dinâmico. A escala possui um máximo de 56 pontos, sendo que a cada tarefa é atribuída uma pontuação de zero a quatro ${ }^{17}$.

$\mathrm{Na}$ avaliação da flexibilidade, foram utilizados três testes de encurtamento muscular: os testes de Thomas normal e alterado e o teste de isquiotibiais. $\mathrm{O}$ teste de Thomas normal avalia o encurtamento do músculo reto femoral, sendo que o teste de Thomas alterado avalia o encurtamento do músculo iliopsoas ${ }^{18}$.

Para análise da marcha foram avaliadas as variáveis velocidade, cadência, comprimento do passo, passada e largura da base de apoio através do protocolo de Cerny, validado no Brasil. Os instrumentos necessários para aplicação do protocolo são: um cronômetro digital, uma passarela de 16 metros e pincéis com cores diferentes ${ }^{19}$.

Para avaliação das amplitudes de movimento, o goniômetro foi posicionado nas articulaçôes envolvidas com o movimento a ser avaliado ${ }^{15}$. Já na avaliação da força muscular, o paciente foi posicionado de forma confortável para execução ativa do movimento, e o avaliador instruiu o paciente a executar o movimento desejado. A pontuação nessa escala foi orientada de acordo com os cinco níveis já descritos nos instrumentos ${ }^{16}$.

Em seguida, procedeu-se a avaliação do equilíbrio corporal utilizando a escala de Berg, na qual o paciente foi instruído a realizar 14 tarefas simples das atividades de vida diárias. Então, o avaliador registrou o escore do paciente em cada item da escala ${ }^{17}$.

Posteriormente, o paciente foi encaminhado para avaliação da flexibilidade feita pelos testes de Thomas e o teste de encurtamento de isquiotibiais. No teste de encurtamento de isquiotibiais, o paciente foi posicionado em decúbito dorsal, e ao comando verbal realizou uma flexão de quadril com o joelho estendido e o tornozelo em posiçáo neutra, ficando o membro contrário em extensão. $\mathrm{O}$ teste foi considerado positivo quando o paciente apresentou grau de flexáo de quadril menor que 70 graus e extensão de joelho menor que zero. Para avaliação do encurtamento dos músculos iliopsoas e reto femoral foi solicitado ao paciente que realizasse flexáo de quadril e de joelho, tracionando o membro inferior contra o tórax, e o avaliador observou se o membro oposto perdeu contato com a mesa. Esse teste pode ser realizado com a perna apoiada na maca ou com a perna pendente fora da maca ${ }^{18}$.

Para a realização do protocolo de Cerny, foram fixados pincéis nos calcanhares dos pacientes para que o chão pudesse ser marcado durante a marcha. As pontas dos pincéis ficaram voltadas para baixo, de modo que a ponta realizasse a marcaçáo do chão. $\mathrm{O}$ avaliador instruiu o paciente a caminhar na sua velocidade habitual de uma extremidade a outra da passarela e, com um cronômetro, registrou o tempo gasto pelo paciente para percorrer os 6 metros centrais da passarela. As medidas entre cada ponto, referente às marcas de contato entre a ponta do pincel e o chão, permitiram avaliar o comprimento do passo, medindo a distância de um pé a outro. O comprimento da passada foi realizado medindo-se a distância entre o mesmo pé, e a cadência foi avaliada medindo o número de passos percorridos em um minuto ${ }^{19}$.

Os participantes foram avaliados por meio de entrevistas (anamnese) e exames laboratoriais e médicos, visando identificar se eles poderiam se submeter à realização de exercícios físicos. O período de treinamento teve duração de 16 semanas, sendo que os sujeitos envolvidos obtiveram a frequência mínima de duas sessóes semanais, com duração de $1 \mathrm{~h}$ por sessão. $\mathrm{O}$ programa de exercício físico acontece em uma sala com disponibilidade de aparelhos de musculaçâo, que na sua maioria são adaptados e com registro de patente pela pesquisadora da Universidade. 
O primeiro mês foi utilizado para adaptação aos aparelhos e, consequentemente, às sessóes, que consistiam em treinamento de resistência (força) intercalado com exercício aeróbio. A cada três exercícios de força eram realizadas atividades de esteira/bicicleta e/ ou cicloergômetro por 10 minutos; realizava-se 30 segundos de descanso entre as séries e de 1 minuto entre os exercícios; a frequência cardíaca máxima (FC máx.) considerada era de $65 \%$, e sucessivamente, realizava-se um total de nove exercícios de força com 25 a 30 minutos de exercício aeróbio, contando com o aquecimento inicial de 5 a 10 minutos, conforme Tabela 1.

Tabela 1: Programa de exercícios físicos realizados pelos pacientes

\begin{tabular}{lc} 
Atividades Sequenciais & Tempo / Repetiçóes \\
\hline Aquecimento & 5 a 10 min \\
\hline Puxador postural & $3 \times 12$ repetiçóes \\
\hline Flexora & $3 \times 12$ repetições \\
\hline Remada cross & $3 \times 12$ repetiçóes \\
\hline $\begin{array}{l}\text { Aeróbio (bicicleta, esteira e/ou } \\
\text { cicloergômetro) }\end{array}$ & \\
\hline Quadríceps e/ou agachamento & $3 \times 12$ repetiçóes \\
\hline Voador & $3 \times 12$ repetições \\
\hline $\begin{array}{l}\text { Panturrilha } \\
\text { Aeróbio (bicicleta, esteira e/ou } \\
\text { cicloergômetro) }\end{array}$ & $3 \times 12$ repetiçóes \\
\hline Bíceps & $3 \times 12$ repetiçóes \\
\hline Extensáo de quadril no solo & $3 \times 12$ repetiçóes \\
\hline Abdominal/abdominal invertido & $3 \times 12$ repetiçóes \\
\hline Alongamento & Livre \\
\hline
\end{tabular}

Fonte: Elaborada pelos autores, 2016.

A mudança para os grupos de hipertensos e diabéticos se deu com a modificação de alguns exercícios atentando para a individualidade de cada um, levando em consideração o local de aplicação da insulina, o volume e a intensidade. Foi necessário atentar para as pessoas com diabetes sobre as informações referentes às partes do corpo onde são aplicadas as injeçôes de insulina, para que não fossem exercitadas. Para os diabéticos insulinodependentes que injetam a insulina no músculo quadríceps, retirou-se os exercícios de cadeira extensora e abdominal, sendo acrescentado nesse grupo outros exercícios como agachamento e abdominal invertido.
Os dados foram analisados com o auxílio do programa estatístico GraphPadPrism 5.0, utilizando como teste estatístico o T de Student não pareado, considerando como nível de significância $\mathrm{p}<0,05$.

\section{Resultados}

O estudo contou com a avaliaçáo de 54 pacientes, 48 do sexo feminino e 6 do sexo masculino, sendo 51 hipertensos, 16 diabéticos e, desses, 13 hipertensos e diabéticos. A idade média dos participantes foi de $61,74+8,62$ para os hipertensos e 60,75+8,72 para os diabéticos. $\mathrm{Na}$ análise do escore da escala de Berg, foi identificada uma média de $53,13+6,21$ pontos no escore de pacientes hipertensos e uma média de 49,31+10,85 pontos no escore de pacientes diabéticos. Em relação à análise estatística para a escala de Berg, foi encontrando um p-valor de 0,1055 , demonstrando que não houve diferença estatística entre os pacientes diabéticos e hipertensos.

No entanto, na avaliação do encurtamento muscular dos hipertensos, após a realizaçáo do teste de Thomas, foi observado que $86 \%(n=44)$ dos participantes apresentaram encurtamento no membro inferior direito e $78 \%(n=40)$ apresentaram encurtamento no membro inferior esquerdo. No teste de encurtamento do músculo reto femoral, cerca de 70,6\% ( $\mathrm{n}=36)$ dos voluntários demonstraram encurtamento no membro inferior direito e 74,7\% ( $\mathrm{n}=33)$ apresentaram encurtamento no membro inferior esquerdo. No teste de encurtamento dos isquiotibiais, 60,8\% ( $n=31)$ dos participantes apresentaram encurtamento no membro inferior direito, e $64,7 \%$ ( $n=33)$, encurtamento no membro inferior esquerdo. Já na avaliação do encurtamento muscular dos diabéticos, na realização do teste de Thomas, foi observado que $81 \%(n=13)$ dos voluntários apresentaram encurtamento no membro inferior direito, e o mesmo número foi encontrado no membro inferior esquerdo. No teste de encurtamento do músculo reto femoral, $68,8 \%$ $(\mathrm{n}=11)$ dos participantes demonstraram encurtamento em ambos os membros inferiores, direito e esquerdo. No teste de encurtamento dos isquiotibiais, $68,8 \%(\mathrm{n}=11)$ dos participantes apresentaram encurtamento no membro inferior direito, e $62,5 \%(n=10)$, encurtamento no membro inferior esquerdo.

$\mathrm{Na}$ análise do Protocolo de Cerny, foram encontrados, para os hipertensos, um tempo médio de 4,12 segundos, uma velocidade média de $1,7 \mathrm{~m} / \mathrm{s}$ e uma cadência média de 119,44 passos. Dessa velocidade média, para as mulheres foi achada uma média de $1,69 \mathrm{~m} / \mathrm{s}$, ao passo que para os homens foi encontrada uma média de $1,77 \mathrm{~m} / \mathrm{s}$. Para os diabéticos, os valores médios encontrados foram de 4,46 segundos para o tempo, $1,37 \mathrm{~m} / \mathrm{s}$ para a velocidade e 108,5 para a cadência. As mulheres 
diabéticas apresentaram uma velocidade média de 1,27 $\mathrm{m} / \mathrm{s}$, e os homens, $1,8 \mathrm{~m} / \mathrm{s}$.

A análise estatística da velocidade da marcha dos pacientes hipertensos e diabéticos não demonstrou diferença significativa com um valor de p igual a 0,078. Os dados da cadência da marcha demonstraram diferença entre os pacientes diabéticos e hipertensos com um valor de p igual a 0,03 . O tempo gasto para percorrer 6 metros entre os pacientes hipertensos e diabéticos também não demonstrou diferença significativa, com $\mathrm{p}=0,38$

Os dados sobre os valores médios da força muscular dos pacientes diabéticos e hipertensos estáo expressos na Tabela 2.

Tabela 2: Valores médios do teste de força muscular (TFM) dos principais grupos musculares nos pacientes diabéticos e hipertensos

\begin{tabular}{lcccc} 
& \multicolumn{2}{c}{ Diabéticos } & \multicolumn{2}{c}{ Hipertensos } \\
Músculo & Direito & Esquerdo & Direito & Esquerdo \\
$\begin{array}{l}\text { Flexores de punho } \\
\text { e dedos }\end{array}$ & 4,85 & 4,69 & 4,80 & 4,82 \\
$\begin{array}{l}\text { Extensores de } \\
\text { punho e dedos }\end{array}$ & 4,56 & 4,56 & 4,49 & 4,65 \\
Tríceps braquial & 4,69 & 4,75 & 4,69 & 4,73 \\
Bíceps braquial & 4,88 & 4,94 & 4,82 & 4,87 \\
Peitoral maior & 4,69 & 4,75 & 4,65 & 4,67 \\
Isquiotibiais & 4,38 & 4,67 & 4,71 & 4,72 \\
Tibial anterior & 4,63 & 4,60 & 4,62 & 4,49 \\
\hline Tríceps sural & 4,69 & 4,53 & 4,74 & 4,7 \\
Quadríceps & 4,88 & 4,80 & 4,92 & 4,88 \\
\hline
\end{tabular}

Fonte: Elaborada pelos autores, 2016.

Os resultados dos valores médios da ADM dos principais movimentos do corpo humano dos pacientes diabéticos e hipertensos estáo demonstrados na Tabela 3 .

Tabela 3: Valores médios da amplitude de movimento articular (ADM) dos principais movimentos do corpo humano nos pacientes diabéticos e hipertensos

\begin{tabular}{lcccc} 
& \multicolumn{2}{c}{ Diabéticos } & \multicolumn{2}{c}{ Hipertensos } \\
$\begin{array}{l}\text { Movimento e } \\
\text { ADM normal }\end{array}$ & Direito & Esquerdo & Direito & Esquerdo \\
$\begin{array}{l}\text { Flexão de joelho } \\
\left(140^{\circ}\right)\end{array}$ & 116,31 & 124,6 & 121,76 & 125,91 \\
$\begin{array}{l}\text { Plantiflexão } \\
\left(45^{\circ}\right)\end{array}$ & 40,25 & 39,6 & 41,98 & 41,49 \\
\hline
\end{tabular}

continua...
Tabela 3: Continuação.

\begin{tabular}{lcccc}
\hline & \multicolumn{2}{c}{ Diabéticos } & \multicolumn{2}{c}{ Hipertensos } \\
$\begin{array}{l}\text { Movimento e } \\
\text { ADM normal }\end{array}$ & Direito & Esquerdo & Direito & Esquerdo \\
$\begin{array}{l}\text { Dorsiflexão } \\
\left(20^{\circ}\right)\end{array}$ & 14 & 11,87 & 13,2 & 12,04 \\
$\begin{array}{l}\text { Extensão de } \\
\text { quadril }\left(10^{\circ}\right)\end{array}$ & 14,8 & 14,93 & 13,87 & 14,09 \\
$\begin{array}{l}\text { Flexão de qua- } \\
\text { dril }\left(125^{\circ}\right)\end{array}$ & 97,25 & 102 & 104,83 & 104,48 \\
$\begin{array}{l}\text { Extensão de } \\
\text { joelho }\left(0^{\circ}\right)\end{array}$ & 0 & 0 & 0 & 0 \\
$\begin{array}{l}\text { Flexão de ombro } \\
\left(180^{\circ}\right)\end{array}$ & 168 & 168,38 & 170,35 & 171,47 \\
$\begin{array}{l}\text { Extensão de } \\
\text { ombro }\left(45^{\circ}\right)\end{array}$ & 44,88 & 51,88 & 47,57 & 48,04 \\
$\begin{array}{l}\text { Flexão de coto- } \\
\text { velo (145 })\end{array}$ & 133,5 & 132,62 & 136,71 & 134,49 \\
$\begin{array}{l}\text { Extensão de } \\
\text { cotovelo }\left(0^{\circ}\right)\end{array}$ & 0 & 0 & 0 & 0 \\
$\begin{array}{l}\text { Flexão de punho } \\
\left(90^{\circ}\right)\end{array}$ & 64,63 & 63 & 60,37 & 60,46 \\
$\begin{array}{l}\text { Extensão de } \\
\text { punho }\left(70^{\circ}\right)\end{array}$ & 58,63 & 62,5 & 58,9 & 61,66 \\
\hline
\end{tabular}

Fonte: Elaborada pelos autores, 2016.

\section{Discussão}

$\mathrm{Na}$ análise dos dados do teste de força muscular (TFM) foi possível observar que os pacientes diabéticos e hipertensos que praticam exercício físico apresentaram um valor ideal de força muscular acima de três pontos, o que demonstrou a eficácia dos exercícios empregados no programa de treinamento utilizado. Isso corrobora com os dados de Silva et al. e Jarek et al., que descrevem, respectivamente, os benefícios da prática de atividade física na terceira idade e a melhora da força muscular e do equilíbrio corporal das pessoas acima de sessenta anos ${ }^{20,21}$.

Dentre as alteraçôes fisiológicas esperadas com o envelhecimento, a diminuição das fibras musculares do tipo II associada ao aumento de massa gorda é a que desencadeia limitaçóes significativas na flexibilidade, força e equilíbrio da pessoa idosa ${ }^{22}$. Nos idosos diabéticos e hipertensos, essas alteraçóes são intensificadas pelo comprometimento da irrigação e da inervação dos músculos esqueléticos ${ }^{23}$. No nosso estudo, foi evidenciada uma diminuição da flexibilidade dos idosos diabéticos e hipertensos, avaliada pelos testes de encurtamento muscular e ADM das articulações. Foi observada uma diminuição significativa da ADM dos movimentos de flexão do ombro, do punho, do quadril, do joelho e do 
tornozelo, corroborando com dados de Shepard et al., que afirmam que os idosos diminuem o nível de flexibilidade na regiáo lombar e no quadril, em torno de 8 a 10 centímetros por década a partir dos 60 anos. No estudo de Pickles et al. foi demonstrado que a pessoa idosa apresenta alteraçóes no padráo flexor de tronco, associado a um aumento da curvatura da coluna torácica e diminuição da lordose lombar, acarretando uma menor estatura e uma posiçáo inclinada do tronco. Essas alteraçôes diminuem a base de equilíbrio da pessoa idosa e desencadeiam maior risco de quedas ${ }^{24,25}$.

Os dados do equilíbrio corporal dos pacientes náo demonstraram diferença significativa entre os grupos de diabéticos e hipertensos. Porém, é possível observar que os pacientes diabéticos apresentaram menores escores na escala de equilíbrio em comparação com os idosos hipertensos. Apesar de os pacientes diabéticos apresentarem escore elevado no teste de força muscular, acredita-se que o comprometimento sensório-motor presente principalmente nos pés desses pacientes contribuiu para a piora do equilíbrio corporal. Cerca de $80 \%$ dos diabéticos apresentam complicaçóes sensório-motoras que modificaram o equilíbrio estático e dinâmico ${ }^{23,26}$. Dessa forma, o equilíbrio corporal não é dependente apenas de um bom controle muscular, mas também de informaçóes sensoriais e proprioceptivas específicas do corpo humano ${ }^{26}$.

Os resultados do Protocolo de Cerny demonstraram que náo ocorreu diferença significativa na velocidade da marcha e no tempo gasto para percorrer os seis metros entre os diabéticos e hipertensos, no entanto, possibilitou a observação de valores menores no grupo de diabéticos. Ao comparar os dados de velocidade da marcha e tempo gasto para percorrer seis metros dos indivíduos com os valores médios de uma população de idosos que não praticam atividade física, como relatado no estudo de Novaes et al., é possível observar valores maiores nos grupos de idosos desse estudo, demonstrando os benefícios do programa de atividade física nessa populaçãa ${ }^{27}$.

Outro parâmetro da marcha analisado neste trabalho foi a cadência, onde foi encontrada diferença significativa entre os grupos $(\mathrm{p}<0,05)$. A menor média de cadência da marcha foi encontrada no grupo dos diabéticos, onde foi evidenciado um maior tempo gasto para percorrer seis metros e menor velocidade da marcha, em comparação com o grupo de hipertensos. No estudo de Fregonesi e Camargo foi demonstrado a relaçáo entre a neuropatia diabética periférica com os déficits na amplitude do passo, velocidade e cadência da marcha, em condições normais (terreno plano, linha reta e velocidade constante), além de déficits de equilíbrio e coordenação motora em condições especiais (aclives, declives e terrenos irregulares). Entretanto, ao comparar os valores médios das cadências da marcha nos grupos deste estudo com valores médios de idosos que não praticam atividade física, é possível observar os benefícios do programa adequado de exercício físico nos grupos de idosos direcionado a esse trabalho ${ }^{27,28}$.

$\mathrm{O}$ avançar da idade gera alteraçóes fisiológicas importantes na populaçáo idosa, e a hipertensão arterial e a diabetes agravam os parâmetros motores, a postura, a flexibilidade, a força muscular e o equilíbrio corporal dessas pessoas. Com base nisso, a realização de estratégias adequadas minimiza esses fatores de risco e promovem bem-estar físico e melhora da qualidade de vida nesses indivíduos.

\section{Conclusão}

O protocolo de exercício físico deve ser direcionado à população alvo e aplicado por um profissional, sendo uma estratégia eficaz no aumento da força muscular, melhora do equilíbrio corporal e dos parâmetros da marcha de idosos hipertensos e diabéticos.

Além disso, foi possível observar que os idosos diabéticos apresentaram um pior desempenho na marcha e equilíbrio corporal, quando comparados com hipertensos, mesmo apresentando um bom nível de força muscular, demonstrando que o equilíbrio corporal e os parâmetros da marcha dependem de outros fatores (sensoriais e proprioceptivos) para o controle motor.

\section{Referências}

1. Instituto Brasileiro de Geografia e Estatística. Síntese de Indicadores Sociais: uma análise das condiçóes de vida da populaçáo brasileira: Rio de Janeiro; 2013 [citado em 2016 jun 10]. Disponível em: <https://goo.gl/qaZyru>.

2. Matsudo SM, Matsudo VKR, Barros Neto TL, Araújo TL. Evolução do perfil neuromotor e capacidade funcional de mulheres fisicamente ativas de acordo com a idade cronológica. Rev Bras Med Esporte. 2003 [citado em 2016 maio 24];9(6):365-76. Disponível em: $<$ https://goo.gl/1VJwFN>.

3. Instituto Brasileiro de Geografia e Estatística. Pesquisa Nacional de Saúde 2013: percepção do estado de saúde, estilos de vida e doenças crônicas. Rio de Janeiro, 2013 [citado em 2016 jun 10]. Disponível em: <https://goo.gl/CEpzKR>.

4. World Health Organization. United Nations high-level meeting on noncommunicable disease prevention and control. New York, 2011 [citado em 2015 set 6]. Disponível em: $<$ https://goo.gl/Ja7Vam>.

5. Diniz AB, Guerra ERFM, Soares RM, Mariz JVB, Cattuzzo MT. Avaliação da cognição, atividade física e aptidão física de idosos: uma revisão crítica. Estud Psicol. 2013 [citado em 2016 jun 12];18(2):315-24. Disponível em: <https://goo.gl/T71bp2>. 
6. Matsudo SMM. Envelhecimento \& atividade física. Londrina: Midiograf; 2001. 195p.

7. Pitanga FJG, Almeida LAB, Freitas MM, Pitanga CPS, Beck CC. Padróes de atividade física em diferentes domínios e ausência de diabetes em adultos. Motricidade. 2010 [citado em 2016 abr 12];6(1):5-17. Disponível em: $<$ https://goo.gl/jknKbD>.

8. Coelho CF, Burini RC. Atividade física para prevenção e tratamento das doenças crônicas não transmissíveis e da incapacidade funcional. Rev Nutr. 2009 [citado em 2016 jul 13];22(6):937-46. Disponível em: $<$ https://goo.gl/4PyLwH >

9. Pedro EM, Bernardes-Amorim D. Análise comparativa da massa e força muscular e do equilíbrio entre indivíduos idosos praticantes e não praticantes de musculação. Conexôes. 2008 [citado em 2016 jun 19];6:174-83. Disponível em: <https:// goo.gl/36rZdT>

10. Böhme MTS. Relaçôes entre aptidão física, esporte e treinamento esportivo. Rev Bras Ci e Mov. 2003 [citado em 2016 set 17];11(3):97-104. Disponível em: <https://goo.gl/z8UAKn>.

11. Franchi KMB, Montenegro Junior RM. Atividade física: uma necessidade para a boa saúde na terceira idade. Rev Bras Promoç Saúde. 2005 [citado em 2016 ago 16];18(3):152-6. Disponível em: $<$ https://goo.gl/yajdda $>$.

12. Mcardle WD, Katch FI, Katch VL. Fisiologia do exercício: energia, nutrição e desempenho humano. $5^{\mathrm{a}}$ ed. Rio de Janeiro: Guanabara Koogan, 2003.

13. Cordeiro J, Del Castillo BL, Freitas CS, Gonçalves MP. Efeitos da atividade física na memória declarativa, capacidade funcional e qualidade de vida em idosos. Rev Bras Geriatr Gerontol. 2014 [citado em 2016 jul 12];17(3):541-52. Disponível em: <https:/goo.gl/gMefVR>.

14. Jenovesi JF, Bracco MM, Colugnati FAB, Taddei JAAC. Evoluçáo no nível de atividade física de escolares observados pelo período de 1 ano. Rev Bras Ci e Mov. 2004 [citado em 2016 maio 13];12(1):19-24. Disponível em: <https://goo.gl/PdRSpe>.

15. Venturini C, Ituassú NT, Teixeira LM, Deus CVO. Confiabilidade intra e interexaminadores de dois métodos de medida da amplitude ativa de dorsiflexão do tornozelo em indivíduos saudáveis. Rev Bras Fisioter. 2006 [citado em 2016 jun 13];10(4):407-11. Disponível em: $<$ https://goo.gl/jomirY>.

16. Junqueira RT, Ribeiro AMB, Scianni AA. Efeitos do fortalecimento muscular e sua relaçáo com a atividade funcional e a espasticidade em indivíduos hemiparéticos. Rev Bras Fisioter. 2004;8(3):247-52. 17. Ferrantin AC, Borges CF, Morelli JGS,
Rebelatto JR. A execução de AVDS e mobilidade funcional em idosos institucionalizados e náo-institucionalizados. Fisioter Mov. 2007 [citado em 2016 set 22];20(3):115-21. Disponível em: <https://goo.gl/stFFz2>.

18. Hoppenfeld S. Propedêutica ortopédica: coluna e extremidades. São Paulo: Atheneu; 1987.

19. Cerny K. A clinical method of quantitative gait analysis: suggestion from the field. Phys Ther. 1983 [citado em 2015 ago 15];63(7):1125-26. Disponível em: <https://goo.gl/ eDkuWH>.

20. Silva NA, Menezes TN, Melo RLP, Pedraza DF. Força de preensão manual e flexibilidade e suas relaçóes com variáveis antropométricas em idosos. Rev Assoc Med Bras 2013 [citado em 2016 jul 13];59(2):128-35. Disponível em: <https://goo. $\mathrm{gl} / 7 \mathrm{wx} 8 \mathrm{bw}>$.

21. Jarek C, Oliveira MH, Nantes WR, Ulbricht L, Mascarenhas LPG. Comparação antropométrica, força muscular e equilíbrio entre idosos praticantes e não praticantes de musculação. Rev Bras Cienc Envelhecimento Hum. 2010 [citado em 2016 maio 24];7(2):173-80. Disponível em: <https:/goo.gl/ARnaTW>.

22. Roberts SB, Young VR, Fuss P, Heyman MB, Fiatarone M, Dallal GE, et al. What are the dietary energy needs of elderly adults? Int. J. Obes Relat Metab Disord. 1992 [citado em 2016 mar 18];16(12):969-74. Disponível em: <https://goo.gl/ QnTFjH $>$.

23. Martinelli AR, Mantovani AM, Nozabieli AJL, Ferreira DMA, Fregonesi CEPT. Alteraçóes dos parâmetros da marcha e déficit sensório-motor associado à neuropatia diabética periférica. Acta Fisiatr. 2014 [citado em 2016 mar 29];21(1):36-40. Disponível em: <https://goo.gl/doQyBa>.

24. Shepard R.J. Aging and exercise. In: Encyclopedia of Sports Medicine and Science. [S.1.]: Internet Society for Sport Science, 1998 [citado em 2016 maio 24]. Disponível em: <https:/goo. $\mathrm{gl} / \mathrm{pHJjbw}>$.

25. Pickles B, Compton A, Cott C, Simpson J, Vandervoort A. Fisioterapia na terceira idade. São Paulo: Santos; 2002.

26. Sales KLS, Souza LA, Cardoso VS. Equilíbrio estático de indivíduos com neuropatia periférica diabética. Fisioter. Pesqui. 2012 [citado em 2015 maio 15];19(2):122-7. Disponível em: $<$ https://goo.gl/cVp8Ph>.

27. Novaes RD, Miranda AS, Dourado VZ. Velocidade usual da marcha em brasileiros de meia idade e idosos. Rev Bras Fisioter. 2011 [citado em 2016 jul 12];15(2):117-22. Disponível em: $<$ https://goo.gl/JWpfyY>.

28. Fregonesi CEPT, Camargo MR. Parâmetros da marcha em portadores de diabetes mellitus. Rev Bras Cineantropom Desempenho Hum. 2010 [citado em 2016 maio 15];12(2):155-63. Disponível em: <https://goo.gl/FVMPL1>.

\section{Como citar este artigo:}

Agostini CM, Rodrigues VS, Guimarães AC, Damázio LCM, Vasconcelos NN. Análise do desempenho motor e do equilíbrio corporal de idosos ativos com hipertensão arterial e diabetes tipo 2. Rev. Aten. Saúde. 2018;16(55):29-35. 